\title{
Raman Optical Activity: A Reliable Chiroptical Technique
}

\author{
Jacques Haesler and Werner Hug*
}

\begin{abstract}
Vibrational optical activity (VOA) is a research field in full expansion. The two main areas where VOA is used are the determination of absolute configurations and the investigation of the secondary and tertiary solution structures of biopolymers. Commercial instrumentation is available since some time for vibrational circular dichroism (VCD) and, more recently, also Raman optical activity (ROA). Here, we describe a recent instrumental implementation of forward scattering, advances in the computational interpretation of $\mathrm{ROA}$, and the determination of the mechanistically important absolute configuration of $(4 E)-3,3-$ dimethyl-5-[(2R,3R)-3-methyloxiran-2-yl] pent-4-en-2-one (1).
\end{abstract}

Keywords: Ab initio computation of ROA - Absolute configuration of a junionone precursor . Graphical interpretation of ROA · Raman optical activity forward scattering · Virtual enantiomer

\section{Introduction}

The spectroscopic methods able todetermine absolute configurations without relying on a material standard of known chirality are anomalous X-ray scattering, various forms of electronic optical activity (EOA), and vibrational optical activity (VOA). There are two manifestations of VOA, namely vibrational circular dichroism (VCD) and Raman optical activity (ROA). The relation between the measured data and the absolute configuration must be made, for all these methods, by computational means.

\footnotetext{
${ }^{*}$ Correspondence: Prof. Dr. W. Hug

University of Fribourg

Department of Chemistry

Chemin du Musée 9

$\mathrm{CH}-1700$ Fribourg

Tel.: +41263008713

Fax: +41263009737

E-mail: werner.hug@unifr.ch

w.hug@gmx.net
}

The relation between microscopic and macroscopic chirality was achieved about half a century ago by anomalous X-ray scattering. ${ }^{11]}$ Measurements of electronic optical activity were of a sufficient precision to likewise accomplish this task, but computations were not, despite the existence of an appropriate theoretical framework. Measurements of vibrational optical activity were unknown. Two failed attempts had been reported for ROA, ${ }^{[2,3]}$ but actual measurements of ROA and VCD became possible only in the $1970 \mathrm{~s} .{ }^{[4-7]}$ The basic theoretical framework of the computation of VOA likewise only was established during this period. Major obstacles had been a lack of an understanding that non-BornOppenheimer terms are decisive for the description of $\mathrm{VCD},{ }^{[8]}$ and that for ROA the consideration of the optical activity tensor alone is insufficient as it is cross terms with the ordinary polarizability tensor that account for ROA's observed size. ${ }^{[9]}$

The feasibility of a reliable determination of an absolute configuration depends on the experimental and computational circumstances. Anomalous X-ray scattering has up to now been considered the gold standard in this area. It requires crystals containing atoms of a heavy element. If they can be grown, which is not always possible, it can still be tricky to pick a representative crystal from a mixture obtained from a sample which is not enantiomerically pure.

Today, despite their late arrival, VCD and ROA are beginning to be recognized as the most effective procedures for determin- ing absolute configurations. In analytical applications, VCD has had an experimental and a computational headstart, but ROA is rapidly catching up. It can deal with smaller sample sizes and has the advantage that the Raman scattering cross section of water is very small. This matters decisively when biopolymers are measured in aqueous solutions. Hydrogen bonding, and the secondary and tertiary structure are aspects which can be investigated with ROA. ${ }^{[10]}$

Progress nothing short of spectacular has happened over a bit more than the past decade in the area of the computation of ROA. ${ }^{[1-14]}$ The ROA spectra of small and medium sized molecules can now reliably be predicted and absolute configurations assigned by the comparison of computed and measured data. This is in marked contrast to EOA where a comparable degree of reliability is still wanting. Tools have also been developed for understanding largescale molecular vibrations ${ }^{[15]}$ and for visualizing how VOA is generated in a vibrating molecule.[16]

\section{Experimental Aspects of ROA}

\subsection{Measurement of ROA}

The invention of the argon ion laser rendered ROA measurements possible about 35 years ago. ${ }^{[4,7]}$ Subsequent instrumental developments turned the barely measurable effect into a reliable chiroptical technique. The main challenges researchers faced were signal degradation by shot noise, by flicker noise, and by 


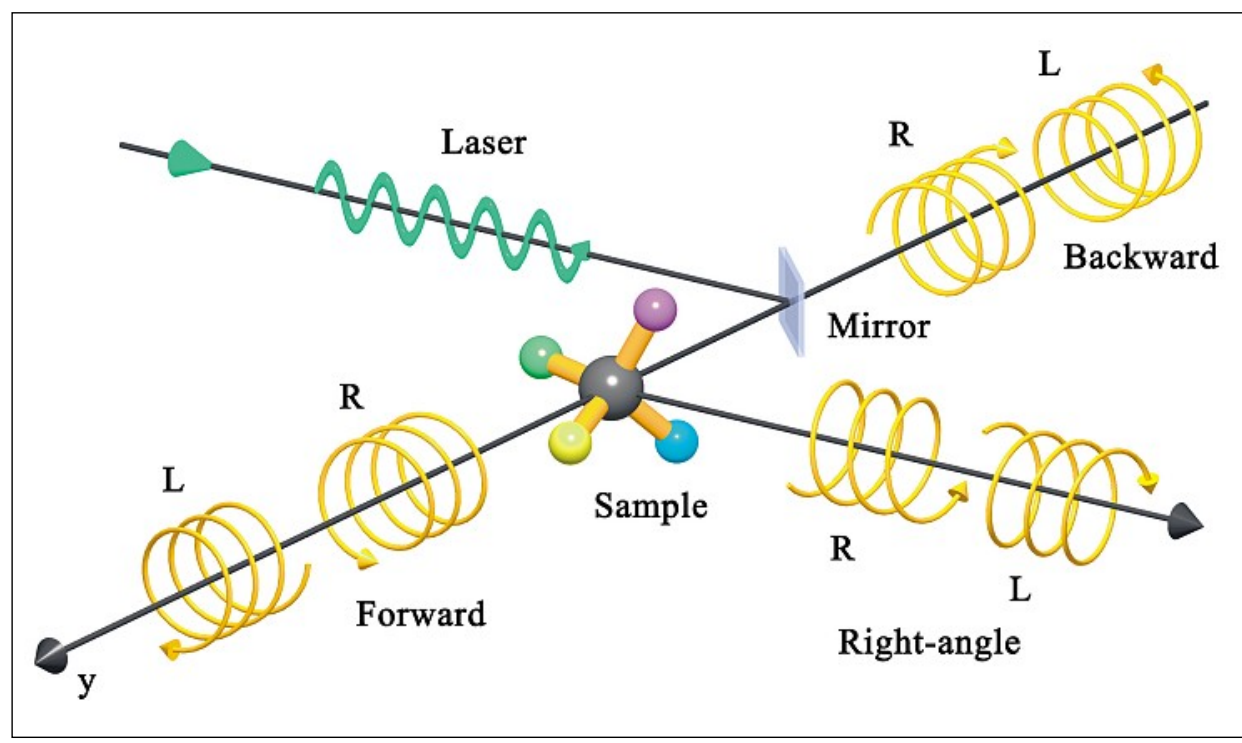

Fig. 1. Backward $(\pi)$, forward $(0)$, and right angle $(\pi / 2)$ scattered circular polarization (SCP) ROA arrangements ( $R$ : right circularly, $L$ : left circularly polarized light).

spurious signals due to optical and other instrumental imperfections.

Shot noise is several orders of magnitude more pronounced in ROA than in ordinary Raman spectroscopy. The remedies are optical multichannel detection, high throughput spectrographs, and efficient collection of the Raman scattered light. The combination of these techniques led to the first instrument capable of recording good quality ROA spectra with acceptable measurement times. ${ }^{[17]}$ The measurements were done in right-angle scattering, a geometry for which spurious signals could decisively be reduced by a dual lens light collection system. ${ }^{[18]}$

Modern ROA spectrometers tend to employ backscattering ${ }^{[19,20]}$ rather than rightangle scattering as it yields higher values for the ratio of the ROA to Raman signal, even though larger sample sizes are required. The use of plane volume phase holographic transmission gratings has led to spectrographs with an étendue larger than can be filled by the Raman scattered light collected from a sample. CCDs provide, for Raman signal levels, shot noise limited performance, and holographic, as well as recently developed dielectric notch and edge filters, have strongly reduced the stray light problem.

The main polarization schemes for recording ROA are incident circular polarization (ICP) and scattered circular polarization (SCP). ${ }^{21]}$ In ICP the polarization of the incident exciting laser beam is modulated between left and right circular, while in SCP the circular polarization content of the scattered light is analyzed. SCP is schematically depicted in Fig. 1 for back-, forward-, and right-angle scattering. SCP has now become the principal method by which ROA spectra are acquired, despite of having originally been considered de- void of practical interest. ${ }^{[22]}$ This is due to the development of a dual arm polarization analyzer which allows the simultaneous recording of right and left circularly polarized light, resulting in an important reduction of flicker noise. ${ }^{[20]}$

The efficient use of SCP in collinear without the solution of the problem of spurious signals. It was found in the form of the virtual enantiomer. ${ }^{[23]}$ The method achieves offset elimination by the subtraction of the ROA spectrum of an optically created enantiomer from the spectrum of the physically present one. The result is the doubling of the desired ROA signal and the elimination of the undesired spurious signals. scattering would not have become possible

\subsection{A Modern ROA Spectrometer with Back- and Forward Scattering Capability}

The original, and up to now sole demonstration of ROA forward scattering was performed in the early nineties with the ICP scheme. ${ }^{[24]}$ The present instrument uses the more exacting SCP scheme. Fig. 2 gives a schematic overview of the instrument. Only the light collection and the polarization analyzing optics for backscattering are shown as the optics for forward scattering is identical, except that it would have to be drawn as the mirror image of that depicted in Fig. 2.

The two arms of the polarization analyzer of the scattered light, at a right angle to each other, can easily be identified in Fig. 2. One branch carries the left and the other the right circularly polarized light, alternately switched between the two arms in order to equalize transmission. The polarization analyzed light exiting the two arms is focused onto the circular ends of a bifurcated fiber optics which combines and transforms the circular cross sections into a single slit image, placed at the entrance of the spectrograph. The spectrograph is a modified version derived from a commercial model based on plane volume phase holographic transmission grating technology.

The virtual enantiomer is created by moving half-wave plates into the optical train before and after the sample, with the motions indicated by horizontal double arrows in Fig. 2. For a detailed description of the four step process we use, the reader is referred to ref. [23]. Linearly polarized components are scrambled by two counterrotating half-wave plates in the exciting and a single one in the scattered light.

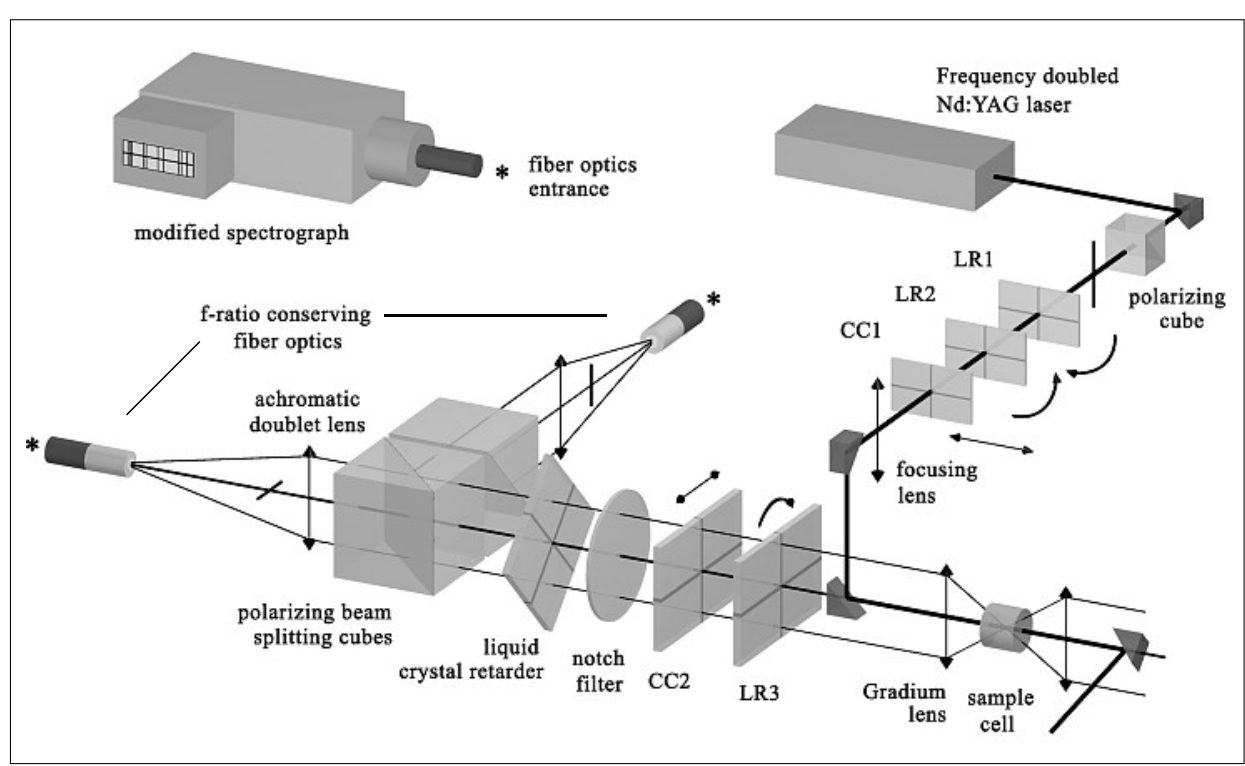

Fig. 2. Schematic overview of the backscattering part of our SCP ROA instrument. The forward scattering optics are not shown here. The two circular fiber optics inputs marked by asterisks are combined and transformed into a single slit image at the entrance of the spectrograph. The direction of rotation of the linear rotators $(L R)$ is indicated by curved arrows while the motion of the circularity converters $(C C)$ corresponds to the horizontal double arrows. 


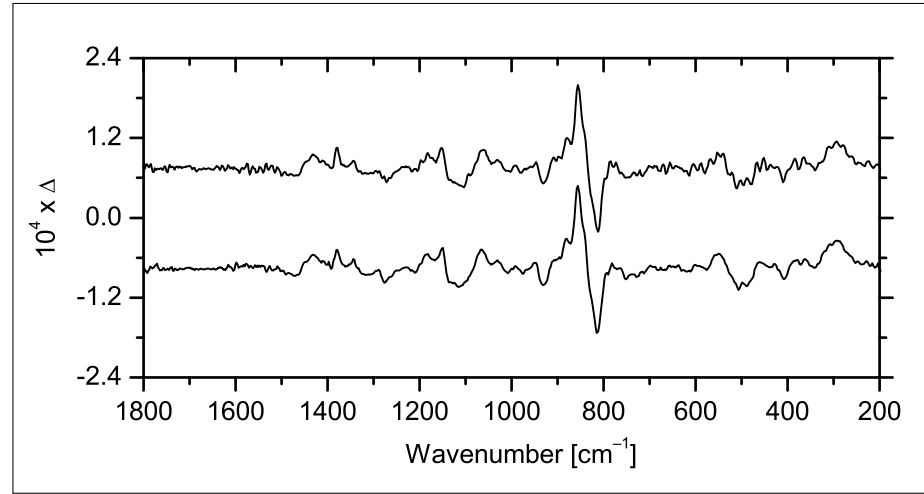

Fig. 3. Comparison of the $\Delta$-curves obtained for epoxyketone 1 in our laboratory in Fribourg in 2006 (top) and in Zürich in 2005 (bottom). Experimental parameters: Top: exposure time: $12 \mathrm{~min}$; laser power at sample: $250 \mathrm{~mW}$. Bottom: exposure time: $25 \mathrm{~min}$; laser power at sample: $500 \mathrm{~mW}$. For both measurements: exciting wavelength: $532 \mathrm{~nm}$; resolution: $7 \mathrm{~cm}^{-1}$; sample size: $35 \mu \mathrm{l}$.

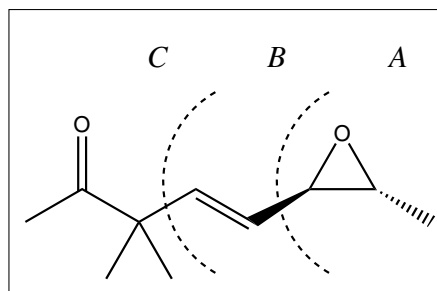

$(R, R)-(E)-1$

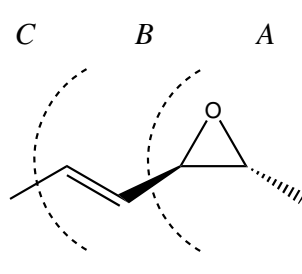

Fig. 4. Structure of the junionone precursor $\mathbf{1}$, and of $\mathbf{2}$ containing the same trans-epoxide moiety. $A, B$ and $C$ represent the groups used in the group coupling matrices (GCM) in Fig 8.

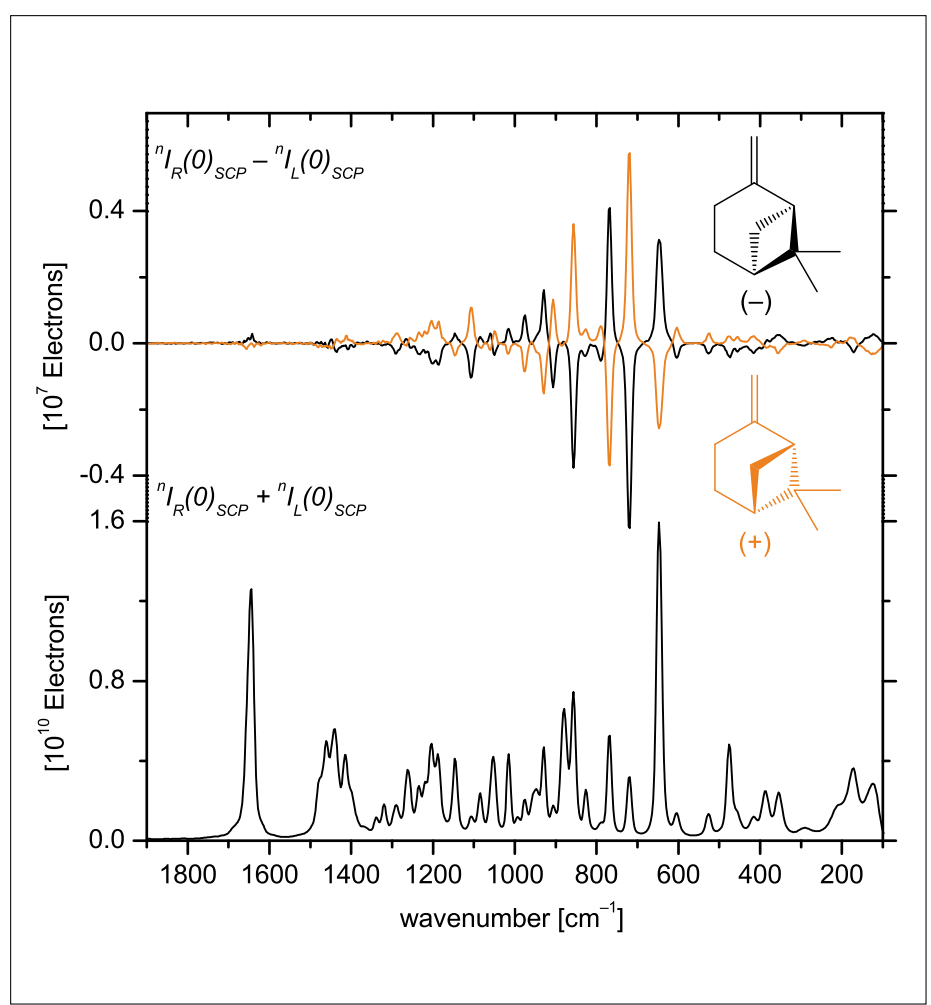

Fig. 5. SCP forward scattering spectra of (+)- $\beta$-pinene (red) and $(-)-\beta$-pinene (black), measured on the Fribourg instrument. Experimental parameters: exposure time: $20 \mathrm{~min}$; laser power at sample: $300 \mathrm{~mW}$; exciting wavelength: $532 \mathrm{~nm}$; resolution: $7 \mathrm{~cm}^{-1}$; sample size: $35 \mu$. The curves are slightly smoothed with a third-order five-point Savitzky-Golay procedure and represent detected photons per CCD column, with one column covering approximately $2.4 \mathrm{~cm}^{-1}$.

\subsection{Comparison of Data Obtained with Different Instruments}

The definitive way of assessing the quality of experimental ROA spectra is the comparison of $\Delta$-curves representing the ratio of the ROA signal to the Raman signal. For backscattering (scattering angle $\pi$ ) the dimensionless $\Delta$ is defined as

$$
\Delta(\pi)=\frac{{ }^{n} N_{R}(\pi)-{ }^{n} N_{L}(\pi)}{{ }^{n} N_{R}(\pi)+{ }^{n} N_{L}(\pi)}
$$

where ${ }^{n} N_{R}(\pi)$ and ${ }^{n} N_{L}(\pi)$ are the numbers of the left $(L)$ and right $(R)$ circularly polarized detected photons for unpolarized $(n)$ exciting light. $\Delta$-Curves are independent of many experimental parameters such as the laser intensity, the measuring time, and the efficiency of the light collection optics, gratings, and detectors. Their measurement is exacting and requires a good signal-to-noise ratio, good stray light control, and clean samples. If these conditions are met, then differences of delta curves measured with different instruments, with a similar resolution and exciting wavelength, will be indicative of instrumental offsets.

Fig. 3 compares the $\Delta$-curve of the epoxyketone 1 (Fig. 4) measured in our laboratory in Zürich in 2005 with that obtained a year later on a newly constructed instrument in Fribourg. The curves demonstrate the reproducibility of ROA data.

It is this high reliability of ROA measurements which has allowed us to determine the absolute configuration of a molecule as subtly chiral as deuterated neopentane.[25]

\subsection{Forward Scattering of (-)- $\beta$-Pinene}

Forward scattering is not expected to become as widely used as backscattering. It is of interest despite of this because it can reveal stereochemically important vibrations which produce little ROA in backward scattering, such as the methylene twisting motion discussed below. The reason for this is that backward scattering is determined by anisotropic components of the ROA scattering tensor, while forward scattering is dominated by isotropic ones. The molecules for which it was first demonstrated are (+)and (-)-trans-pinane and (-)- $\beta$-pinene. ${ }^{[24]}$ Though the $(+)$-enantiomer of $\beta$-pinene could not be measured - it was not commercially available at that time - the mirror image spectra obtained for the enantiomers of trans-pinane lend credibility also to the data obtained for (-)- $\beta$-pinene.

The SCP forward scattering spectra of (+)- and (-)- $\beta$-pinene, measured on the
Fribourg instrument in a precision cell, are shown in Fig. 5. The curves are essentially mirror images of each other which is a strong indication of the absence of spurious signals.

SCP data were also measured in small capillaries, with identical results. They are compared in Fig. 6 with the historic spectrum of (-)- $\beta$-pinene measured with ICP. The signal-to-noise ratio of the recent spectra is visibly better despite the fact that they were obtained with a much smaller sample size and with only half of the laser power in a third of the measurement time expended on the historic spectrum.

A main discrepancy between the old and new data exists, though, namely for the highly polarized band at $652 \mathrm{~cm}^{-1}$. Before the advent of advanced offset compensation schemes, ${ }^{[23]}$ such bands could simply not be reliably measured in collinear scattering the only way was in right angle scattering with our dual lens light collection system. ${ }^{[18]}$ The general experience was that strongly polarized bands show negligible ROA so that their presence was used to null instrumental offset. Clearly, as our precise data now demonstrate, this procedure was not justified for forward scattering. The ROA of the $652 \mathrm{~cm}^{-1}$ band of $\beta$-pinene is, for this scattering geometry, far from negligible. For 

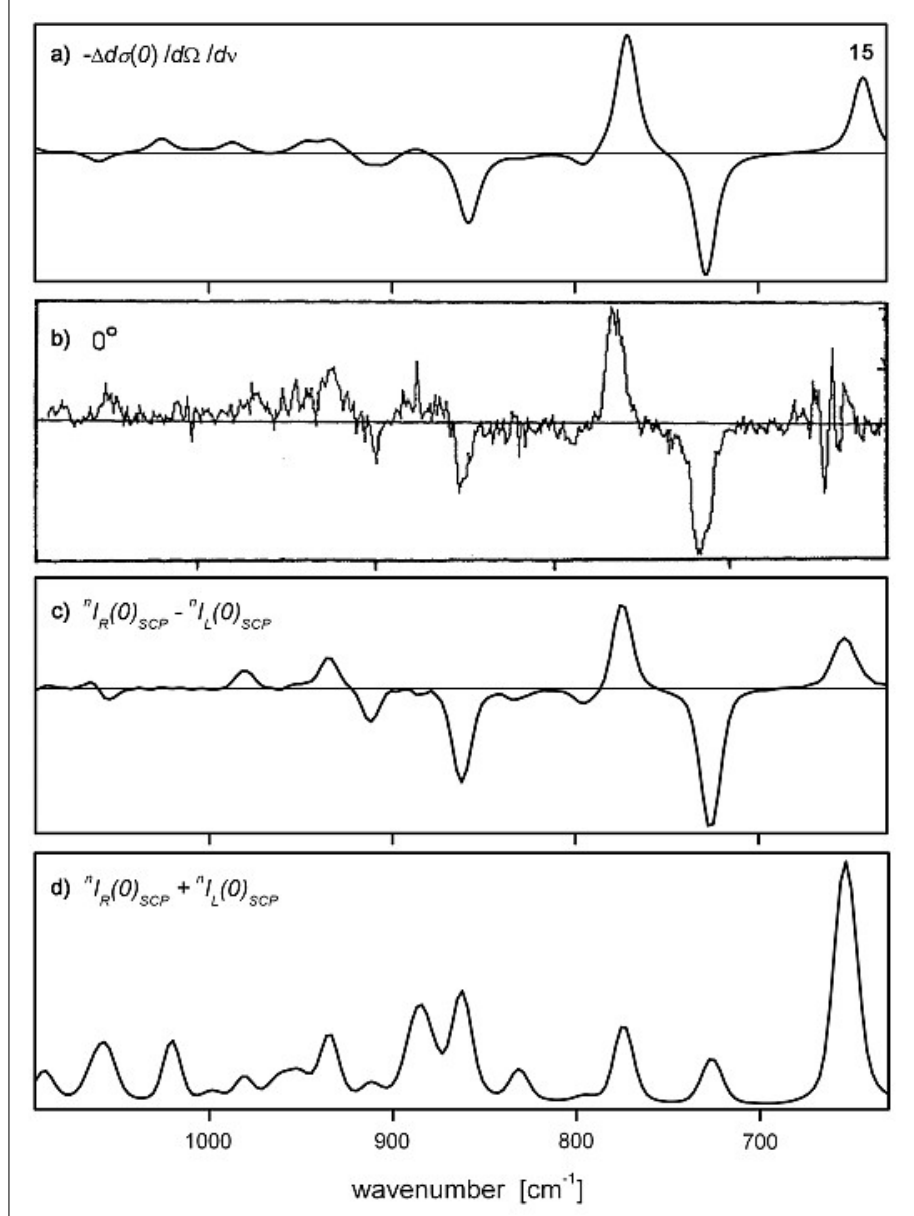

Fig. 6. Recent disposable capillaries Raman and ROA forward scattering spectra of (-)- $\beta$-pinene compared to older measurements and $a b$ initio computations. a) computed ROA; computational parameters: see text. b) reproduction of the ROA spectrum measured by Barron et al. in 1990.[24] c) ROA spectrum, and d) Raman spectrum measured on the Fribourg instrument in a capillary with a $8.5 \mu \mathrm{l}$ sample size. Exposure time: 20 min; laser power at sample: $250 \mathrm{~mW}$; other measurement parameters: as for Fig. 5. backscattering, which is not shown here, the ROA signal is considerably smaller but it does not completely vanish either. ${ }^{[26]}$

$A b$ initio calculations at the DFT/ B3LYP/6-311++G** level with Gaussian03 ${ }^{[27]}$ for the force field and the TDHF/ rDPS level with Dalton ${ }^{[28]}$ for the electronic tensors $^{[12]}$ indicate that the band is due to vibration 15 , a pinane ring stretching mode, coupled simultaneously with a $\mathrm{C}=\mathrm{CH}_{2}$ methylene twisting motion and an in-phase stretching motion of the molecule's two methyl groups with respect to the carbon atom to which they are bound. A detailed analysis of the quasi-atomic contribution pattern (ACP) shows that it is the methylene twist which gives rise to most of the isotropic ROA scattering. ${ }^{[26]}$ The methylene twisting motion also makes the dominant contribution to vibration 16 which has the largest ROA in the spectrum. ${ }^{[24]}$ The phase of the coupling with the motion of the remainder of the molecule is largely opposite to that observed for vibration 15 . This explains the opposite signs of the ROA of vibration 15 and 16.

\section{Art of Understanding ROA}

The interpretation of vibrational optical activity requires the understanding of the vibrational motions of the nuclei of a molecule as well as of its electronic structure. At first sight, this appears to render its computation more difficult and fraught with uncertainty than that of electronic optical activity. In practice, the ab initio computation of VOA has turned out far more reliable than that of EOA, while the generalization of the information obtained from computed numerical results is indeed more difficult.

The explanation of these observations derives from the fact that the shape of the vibrations is more important in VOA than the electronic structure of the molecule. The $a b$ initio computation of molecular force fields, in turn, is easier than the computation of the excited electronic states, which, implicitly or explicitly, determine the various manifestations of EOA.

Two newly developed tools have proved helpful for solving the interpretational problem of VOA. One is an atomistic approach for visualizing how VOA is generated in a vibrating molecule, ${ }^{[16]}$ the other a novel way to compare and decompose the vibrational motions of the nuclei of a molecule. ${ }^{[15]}$ We will limit the discussion in the following to ROA. The extension to VCD is obvious. [29]

The way in which the vibrations of a molecule and its electronic structure matter separately for the ROA generated by a vibrating molecule is best seen from the theoretical expressions of the scattering cross section $\sigma=\sigma_{L}+\sigma_{R}$ for Raman and $\Delta \sigma=\sigma_{L}-\sigma_{R}$ for ROA scattering. We assume the scattered circular polarization (SCP) scheme described in the preceding experimental section. For the differential scattering cross sections $d \sigma$ and $\Delta d \sigma$ of a molecule, averaged over all of the molecule's spatial orientations, one then has, for backward $(\pi)$ and forward $(0)$ scattering into an infinitesimal element $d \Omega$ of the solid angle $\Omega^{[30]}$

$$
\begin{gathered}
d \sigma(\pi)=d \sigma(0)=d \sigma_{L}+d \sigma_{R}= \\
K\left(90 a^{2}+14 \beta^{2}\right) d \Omega, \\
-\Delta d \sigma(\pi)=-\left(d \sigma_{L}(\pi)-d \sigma_{R}(\pi)\right)= \\
\frac{4 K}{c}\left(12 \beta_{G}^{2}+4 \beta_{A}^{2}\right) d \Omega,
\end{gathered}
$$

$$
\begin{aligned}
-\Delta d \sigma(0)= & -\left(d \sigma_{L}(0)-d \sigma_{R}(0)\right)= \\
& \frac{4 K}{c}\left(90 a G^{\prime}+2 \beta_{G}^{2}-2 \beta_{A}^{2}\right) d \Omega,
\end{aligned}
$$

$$
K=K_{p}=\frac{1}{90}\left(\frac{\mu_{0}}{4 \pi}\right)^{2} \omega_{0} \omega_{p}^{3},
$$

where $\mu_{0}$ is the permeability of the vacuum and $\omega_{0}$ and $\omega_{\mathrm{p}}$ are the pulsations of the exciting and the scattered light, respectively. The minus sign has been introduced in order to render the standard definition of left minus right for optical activity compatible with the custom of representing experimental ROA spectra as right minus left.

$a^{2}$ and $\beta^{2}$ are the isotropic and anisotropic invariant, respectively, due to the ordinary electric dipole-electric dipole Raman scattering tensor $\boldsymbol{a}$, and $a G^{\prime}$ the isotropic, and $\beta_{G}^{2}$ and $\beta_{A}^{2}$ the anisotropic invariants which stem, respectively, from the cross terms of $\boldsymbol{a}$ with the optical activity tensor $\boldsymbol{G}$ ' and with $\boldsymbol{A}$, the electric dipole-electric quadrupole polarizability tensor. The expressions are assumed to pertain to individual vibrations $p$ with the vibration explicitly specified only in the definition of the constant $K_{p}$.

In the Placzek polarizability theory[31] the various transition tensors $\boldsymbol{T}_{f \leftarrow i}$ (where $\boldsymbol{T}$ stands for $\boldsymbol{a}, \boldsymbol{G}$ ' or $\boldsymbol{A}$ ), which give rise to the above invariants, for the molecule transiting between an initial vibrational state $i$ and a final state $f$ for vibration $p$, assumed here to be harmonic, are written as

$$
\boldsymbol{T}_{f \leftarrow i} \approx\left\langle f\left|\boldsymbol{T}^{e}\right| i\right\rangle \approx\left(\frac{\partial \boldsymbol{T}^{e}}{\partial Q_{p}}\right)_{0}\left\langle f\left|Q_{p}\right| i\right\rangle,
$$


where $\boldsymbol{T}^{e}$ is a purely electronic tensor. We notice that terms independent of the normal coordinate $Q_{p}$ on the right hand side vanish because of the orthogonality of the vibrational wavefunctions $\langle f|$ and $\langle i|$.

Eqn. (3), when expressed in Cartesian derivatives, can form the basis of an atomistic decomposition of the scattering cross sections as all invariants occurring in Eqns (2) can be expressed as sums over monoand dinuclear terms. ${ }^{[16]}$ If $I_{p}$ stands for any of the invariants occurring in Eqns (2), one can write

$$
I_{p}=\left\langle f\left|Q_{p}\right| i\right\rangle^{2} \sum_{a, b} J_{a b, p},
$$

where the sums over $a$ and $b$ are over a molecule's nuclei. If we exemplify the form of the local invariant expressions $J_{a b, p}$ by $\beta_{G_{a b, p}}^{2}$ we have

$$
\begin{aligned}
J_{a b, p}=\beta_{G_{a b, p}}^{2} & =\frac{1}{2} \sum_{\mu, v} \sum_{i, j}\left[3\left(\frac{\partial \alpha_{\mu \nu}^{e}}{\partial x_{a i}}\right)_{0}\left(\frac{\partial G_{\mu \nu}^{e}}{\partial x_{b j}}\right)_{0}\right. \\
& \left.-\left(\frac{\partial \alpha_{\mu \mu}^{e}}{\partial x_{a i}}\right)_{0}\left(\frac{\partial G_{\mu \mu}^{e}{ }^{\prime}}{\partial x_{b j}}\right)_{0}\right] L_{a i, p}^{x} L_{b j, p}^{x}
\end{aligned}
$$

The numerical results of such a decomposition can be represented graphically in the form of a matrix. It is often convenient to collect the nuclei of several atoms into a group, which yields the group coupling matrices (GCMs). ${ }^{[16]}$ The use of such GCMs is shown in the following section.

The direction and relative size of the motion of nucleus $a$ in vibration $p$ about the equilibrium position is characterized by the vector $\boldsymbol{L}_{a, p}^{x}=\boldsymbol{L}_{a, p} / \mathrm{V}_{a}$, with $\boldsymbol{L}_{a, p}$ the eigenvectors of the vibrational Eigenvalue problem. Traditionally, vibrational motions have been compared by their representation in internal coordinates. For sizable molecules this becomes unwieldy. We have developed a procedure ${ }^{[15]}$ which allows the direct comparison of normal coordinates, including translations and rotations, as vectors in $3 N$ dimensional space, where $N$ is the number of nuclei considered. The part $\boldsymbol{L}_{p}^{A}$ of a normal mode $p$ located on a fragment $A$ with $N_{A}$ nuclei can be written as

$$
\boldsymbol{L}_{p}^{A}=\sum_{a=1}^{N_{A}} \sum_{i=1}^{3} L_{a i, p}^{A} \boldsymbol{e}_{a i}^{A},
$$

where the basis $\left\{\boldsymbol{e}_{a i}^{A}\right\}$ consists of Cartesian unit vectors (index $i$ ) located on the $N_{A}$ nuclei (index $a$ ) of the fragment $A$. If the nuclei of two similar fragments $A_{1}$ and $A_{2}$, each with $N_{A}$ nuclei, of two molecules
1 and 2 are properly superposed, then the parts of two normal modes $p$ and $p$ ' located on $A_{1}$ and $A_{2}$, respectively, can be meaningfully compared by double-contracting the dyads $\boldsymbol{L}_{p}^{A_{2}} \boldsymbol{L}_{p}^{A_{2}}$, and $\boldsymbol{L}_{p}^{A_{l}} \boldsymbol{L}_{p}^{A_{l}}$. This allows for a quantitative definition of the overlap $O$ of the nuclear motions in $p$ and $p$ ' on the two fragments

$$
O_{p^{\prime} p}^{A_{2} A_{1}}=\boldsymbol{L}_{p^{\prime}}^{A_{2}} \boldsymbol{L}_{p^{\prime}}^{A_{2}}: \boldsymbol{L}_{p}^{A_{1}} \boldsymbol{L}_{p}^{A_{1}}
$$

where the double-dot product is defined by

$$
\boldsymbol{L}_{s} \boldsymbol{L}_{t}: \boldsymbol{L}_{u} \boldsymbol{L} \boldsymbol{v}_{t}=\left(\boldsymbol{L}_{s} \cdot \boldsymbol{L}_{u}\right)\left(\boldsymbol{L}_{t} \cdot \boldsymbol{L}_{v}\right)
$$

For the similarity $S$ one can write

$$
\begin{aligned}
& S_{p^{\prime} p}^{A_{2} A_{1}}=\frac{O_{p^{\prime} p}^{A_{2} A_{1}}}{\left|\boldsymbol{L}_{p^{\prime}}^{A_{2}} \boldsymbol{L}_{p^{\prime}}^{A_{2}} \| \boldsymbol{L}_{p}^{A_{1}} \boldsymbol{L}_{p}^{A_{1}}\right|} \\
& =\frac{\boldsymbol{L}_{p^{\prime}}^{\prime 2} \boldsymbol{L}_{p^{\prime}}^{\alpha_{2}}: \boldsymbol{L}_{p}^{*_{1}} \boldsymbol{L}_{p}^{\mu_{1}}}{\sqrt{\boldsymbol{L}_{p^{\prime}}^{A_{2}} \boldsymbol{L}_{p^{\prime}}^{A_{2}}: \boldsymbol{L}_{p^{\prime}}^{A_{2}} \boldsymbol{L}_{p^{\prime}}^{A_{2}}} \sqrt{\boldsymbol{L}_{p}^{A_{1}} \boldsymbol{L}_{p}^{A_{1}}: \boldsymbol{L}_{p}^{A_{1}} \boldsymbol{L}_{p}^{A_{1}}}}
\end{aligned}
$$

$O$ and $S$ can vary between 0 and 1 , with $O$ being dependent and $S$ independent of the fraction of a normal mode located on the fragment one considers.

Aligning the fragments $A_{1}$ and $A_{2}$ can be done by a least-square fitted quaternion rotation chosen so that the sum of the squares of the mass-weighted distances between nuclei is minimal.

Alternative ways to use the procedure are to compare the shape of the same mode $p$ on different fragments $A$ and $B$ of a single molecule, or to compare different modes $p$ and $p$ ' of the same molecule on the same fragment $A$.

For a discussion of how local translational and rotational motion can be subtracted for nuclear motions on individual fragments, see ref. [15].

\section{Determination of an Absolute Configuration}

The trans-epoxyketone $\mathbf{1}$ (Fig 4a) is a key intermediate in the synthesis ${ }^{[32]}$ of junionone, a molecule with interesting olfactory properties. The synthesis occurs with significant epimerization, but a reasonable enantiomeric excess of either of the two enantiomers of junionone can be obtained by the choice of the Lewis acid used to activate the epoxide moiety of $\mathbf{1}$. Thus, while the absolute configuration of junionone itself was known, ${ }^{[33]}$ ascertaining the absolute configuration of $\mathbf{1}$ was decisive for elucidating the detailed mechanism of the synthetic pathway. A sample of pure $(R, R)-(E)-\mathbf{1}$ was therefore submitted for ROA spectroscopy, and the absolute configuration was determined by the comparison of the measured spectrum and the $a b$ initio computed spectrum of the equilibrium mixture of the six most abundant conformers of $\mathbf{1}$.

Fig. 7 compares the measured and computed data. The agreement is remarkable despite the fact that the computations were done for the isolated conformers while the measurements are performed for the liquid phase. It leaves no doubt about the $(2 R, 3 R)$ absolute configuration of the synthetic compound. The spectra also demonstrate conclusively the overwhelming advantage VOA has, as compared to EOA, through the presence of dozens of optically active bands, rather than of a few at best experimentally accessible electronic states.

The salient experimental ROA feature is a positive band at $858 \mathrm{~cm}^{-1}$. It is due to vibration 26 , and its discussion allows us to exemplify important aspects of the interpretation of ROA spectra. Fig. 8 shows the vibrational energy distribution of vibration 26 for the dominant $(50 \%)$ conformer of 1. This vibration extends over most of the molecule and not just over the strongly dissymmetric epoxide moiety.

In order to gain insight into the generation of the ROA of vibration 26, it is useful to divide the molecule into groups, and to inspect the group coupling matrices. The three chosen groups are the dissymmetric trans-epoxide moiety (A), the $\mathrm{C}=\mathrm{C}$ double bond with its two attached hydrogen atoms (B), and the remainder of the molecule bound to the double bond $(\mathrm{C})$.

The GCM for the main conformer is represented in Fig. 8. It shows that most of the positive ROA of vibration 26 is generated by the coupling between $\mathrm{A}$ and $\mathrm{C}$, and only a modest part within the trans-epoxide moiety A. Likewise, little ROA is generated within $\mathrm{C}$, despite the strongly chiral geometry which this achiral fragment assumes in the dominant conformer of 1 . These findings disagree with the short range character assumed for ROA in the older literature. ${ }^{[34,35]}$ They confirm, on the other hand, the observation we have made earlier for Galaxolide, namely that longer range interactions cannot be neglected. [36]

The ROA generated by the interaction of two fragments separated by a $\mathrm{C}=\mathrm{C}$ bond raises the question if the absolute configuration of $\mathbf{1}$ can be determined by the comparison of its ROA with that of smaller molecules containing the trans-epoxide moiety A. ${ }^{[37]}$ We will consider 2 as an example. Its computed ROA spectrum is compared in Fig. 9 with that of the dominant conformer of $\mathbf{1}$. Vibration 13 of $\mathbf{2}$ is calculated to occur in the same spectral range as vibration 26 of 1 . On the fragment $A$ the similarity of the two vibrations, as defined by Eqn. (9), reaches 0.91 . From the GCM of vibration 13 (Fig. 8) it transpires that its ROA 


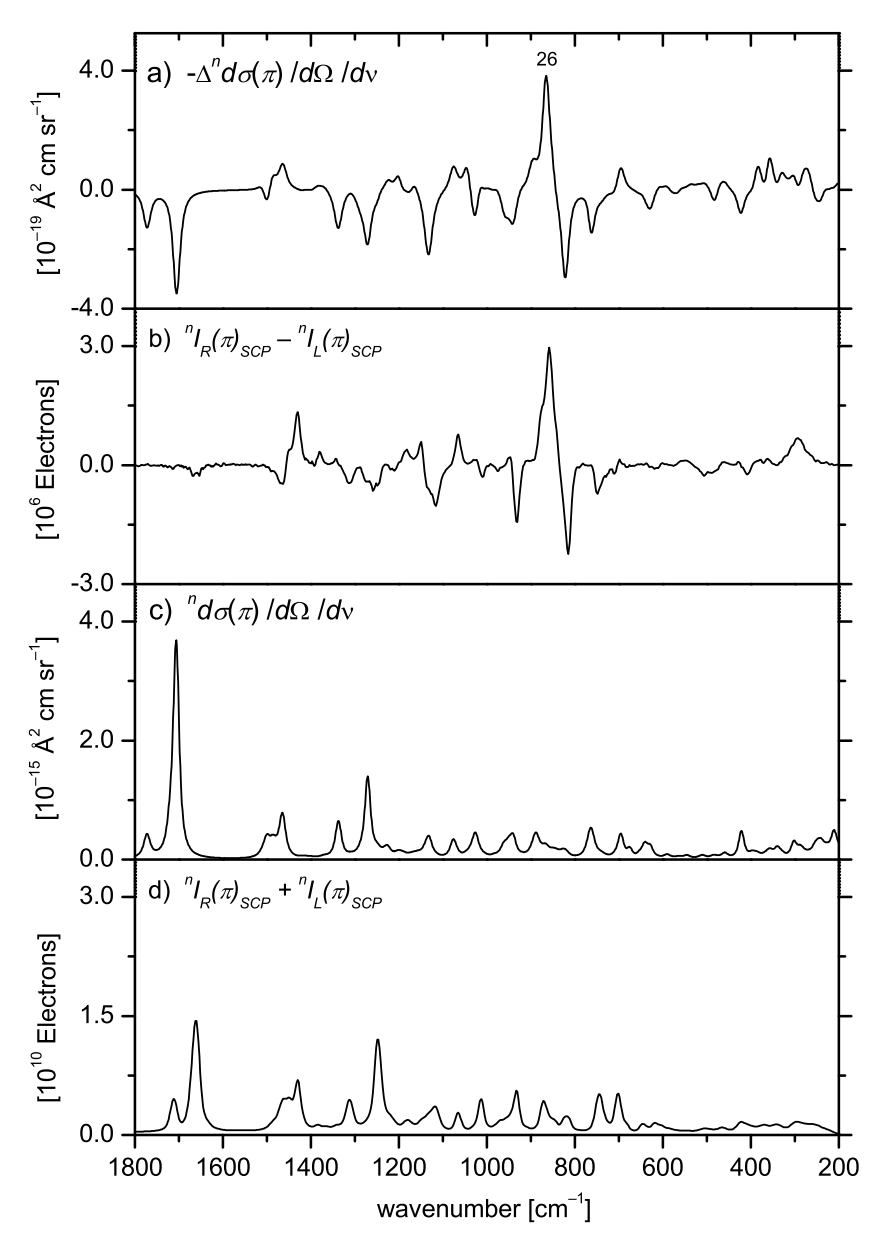

Fig. 7. Computed and measured Raman and ROA spectra of the junionone precursor 1. a) computed ROA, c) computed Raman spectrum; computational parameters as for $\beta$-pinene, see text. b) ROA, and d) Raman spectrum measured on the Zürich instrument. Exposure time: $25 \mathrm{~min}$; laser power at sample: $500 \mathrm{~mW}$; sample size: $35 \mu \mathrm{l}$. Other measurement parameters: as in Fig. 5.

is determined by the trans-epoxide moiety $A$, with the same positive sign as in the epoxiketone 1 . As the overall sign of vibration 26 of $\mathbf{1}$ is not determined by fragment $A$ alone but rather by the interaction between $A$ and $C$, the agreement of the signs of the ROA of vibrations 26 and 13 is somewhat fortuitous.

The situation is far more favorable for pairs of vibrations of the two molecules which are better localized on the common trans-epoxide moiety than vibration 26 is. The identification of such vibrations cannot be done, though, by the mere comparison of spectra but requires computational support. The experience is that, with this support at hand, it is entirely possible to establish, based on ROA spectra, the relative configuration of a series of molecules containing the same chiral fragment.

\section{Outlook}

New analytical technologies which require new skills inevitably face a time delay until they are accepted as mainstream meth-

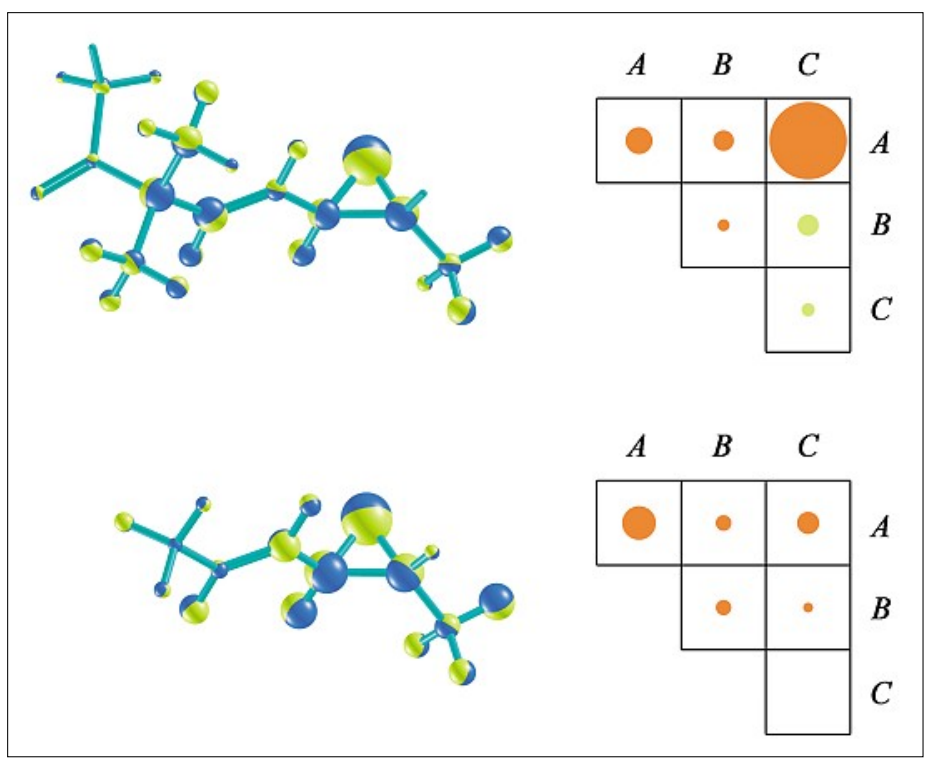

Fig. 8. Representation of vibration 26 of 1 (top) and vibration 13 of 2 (bottom). The volume of the spheres is proportional to the vibrational energy, with the direction of motion indicated by shading. The ROA group coupling matrices are drawn to the right with the groups $A, B$, and $C$ defined in Fig. 4. Pictures were drawn with VOAView, ${ }^{[26]}$ a visualization program written in Matlab.

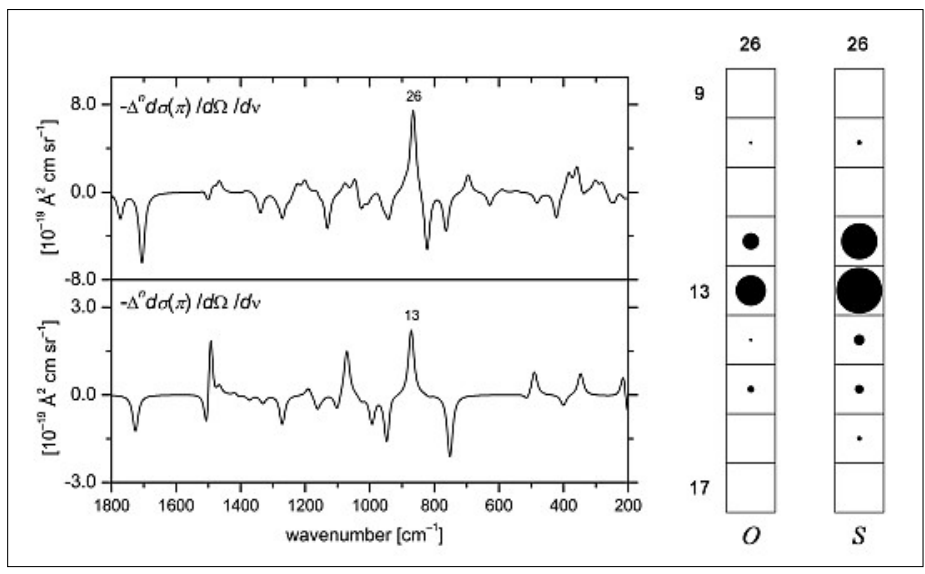

Fig. 9. Computed ROA spectra of the main conformers of $\mathbf{1}$ and 2. The overlap $(O)$ and similarity $(S)$ of vibration 26 of $\mathbf{1}$ with the vibrations of a similar energy of 2 are indicated to the right, with the surface of circles proportional to the values. A circle which fills its square means a value of $1 .[38]$

ods. This is so even if they are potentially far more powerful than the established techniques in their field. Vibrational optical activity, and in particular Raman optical activity, belong in this category. While ROA spectra could be recorded quite reliably by specialized research groups, doing such measurements as a routine task in an analytical setting was, until recently, out of question. This has changed with the commercial availability of reliable instrumentation capable of measuring sub-mg sample quantities.

The extraction of absolute configurations from ROA spectra does require skills not automatically part of a stereochemist's toolbox. Few empirical rules are yet avail- able, and the reliable approach is the $a b$ initio computation of the stereochemically relevant structural part of a molecule. The good news is the availability of quantum chemistry programs which can be used for this task without the need of a detailed understanding of their elaborate algorithms, in precisely the same way a spectrometer is used for recording spectra, and the availability of powerful graphical methods for understanding computed results. There is little doubt that Raman optical activity will become, over the coming decade, an important mainstream method for determining the absolute configuration, and the solution conformations, of chiral molecules. 


\section{Acknowledgement}

The authors are indebted to Dr. Martin Lovchik for a sample of (4E)-3,3-dimethyl5 -[(2R,3R)-3-methyloxiran-2-yl]pent-4-en-2one devoid of contamination by the (4Z) and cis-epoxy isomers. ${ }^{[37]}$ The computations were done on the Opteron cluster of the University of Fribourg. The project was supported through SNF grants 200020-103750 and 200020-112201.

Received: February 15, 2008

[1] J. M. Bijvoet, A. F. Peerdeman, A. J. Bommel, Nature 1951, 168, 271.

[2] A. Kastler, C.R. Acad. Sci. Paris 1930, 191, 565.

[3] S. Bhagavantam, S. Venkateswaran, Nature 1930, 125, 237.

[4] L. D. Barron, M. P. Bogaard, A. D. Buckingham, J. Am. Chem. Soc. 1973, 95, 603.

[5] G. Holzwarth, E. C. Hsu, H. S. Mosher, T. R. Faulkaner, A. Moscowitz, J. Am. Chem. Soc. 1974, 96, 251.

[6] L.A. Nafie, J. C. Cheng, P. J. Stephens, J. Am. Chem. Soc. 1975, 97, 3842.

[7] W. Hug, S. Kint, G. F. Bailey, J. R. Scherer, J. Am. Chem. Soc. 1975, 97, 5589.

[8] L. A. Nafie, T. H. Walnut, Chem. Phys. Lett. 1977, 49, 441.

[9] L. D. Barron, A. D. Buckingham, Mol. Phys. 1971, 20, 1111.

[10] L. D. Barron, F. Zhu, L. Hecht, G. E. Tranter, N. W. Isaacs, J. Mol. Struct. 2007, 434,7 .
[11] T. Helgaker, K. Ruud, K. L. Bak, P. Joergensen, J. Olsen, Faraday Discuss. 1994, 99, 165.

[12] G. Zuber, W. Hug, J. Phys. Chem. A 2004, 108, 2108.

[13] K. J. Jalkanen, R. M. Nieminen, M. Knapp-Mohammady, S. Suhai, Int. J. Quantum Chem. 2003, 92, 239.

[14] V. Liégeois, K. Ruud, B. Champagne, J. Chem. Phys. 2007, 127, 204105.

[15] W. Hug, M. Fedorovsky, Theoret. Chem. Acc. 2008, 119, 113.

[16] W. Hug, Chem. Phys. 2001, 264, 53.

[17] W. Hug, A. Kamatari, K. Srinivasan, H.-J. Hansen, H.-R. Sliwka, Chem. Phys. Lett. 1980, 76,469 .

[18] W. Hug, Appl. Spectosc. 1981, 35, 115.

[19] L. D. Barron, L. Hecht, W. Hug, Chem. Phys. Lett. 1989, 158, 341.

[20] W. Hug, G. Hangartner, J. Raman Spectrosc. 1999, 30, 841.

[21] L. Hecht, D. Che, L. A. Nafie, J. Phys. Chem. 1992, 96, 4266.

[22] L. Hecht, L. A. Nafie, Mol. Phys. 1991 , $72,441$.

[23] W. Hug, Appl. Spectrosc. 2003, 57, 1 .

[24] L. D. Barron, L. Hecht, A. R. Gargaro, W. Hug, J. Raman. Spectrosc. 1990, 21, 375.

[25] J. Haesler, I. Schindelholz, E. Riguet, C. G. Bochet, W. Hug, Nature 2007, 446, 526.

[26] J. Haesler, PhD Thesis University of Fribourg, Switzerland No. 1509, 2006.

[27] M. J. Frisch, et al. Gaussian 03 Revision C.02, 2004, Gaussian Inc., Wallingford, Connecticut.
[28] Dalton, a molecular electronic structure program, release 1.1, 2000; see: http:// www.kjemi.uio.no/software/dalton/dalton. html.

[29] W. Hug, J. Haesler, Int. J. Quantum Chem. 2005, 104, 695.

[30] W. Hug, in 'Handbook of Vibrational Spectroscopy', Ed. J. M. Chalmers, P.R. Griffiths, John Wiley \& Sons Ltd, Chichester, 2002, p.745.

[31] G. Placzek, in 'Handbuch der Radiologie', Akad. Verlagsges. Leipiz, 1934, p. 205.

[32] M. A. Lovchik, G. Frater, A. Goeke, W. Hug, Chem. Biodiversity 2008, 5, 126.

[33] A. F. Thomas, M. Ozainne, Chem. Commun. 1973, 19, 746.

[34] a) L. D. Barron, L. Hecht, A. F. Bell, G. Wilson, Appl. Spectrosc. 1996, 50, 619; b) L. D. Barron, L. Hecht, E. W. Blanch, A. F. Bell, Progr. Biophys. Mol. Biol. 2000, $73,1$.

[35] L. A. Nafie, Appl. Spectrosc. 1996, 50, 14.

[36] G. Zuber, W. Hug, Helv. Chim. Acta 2004, 87, 2208.

[37] M. A. Lovchik, PhD Thesis University of Zürich, Switzerland, 2006.

[38] M. Fedorovsky, PyVib2, a program for analyzing vibrational motion and vibrational spectra, 2007, http://pyvib2. sourceforge.net. 